\title{
A Universal Fast Colorimetric Method for DNA Signal Detection with DNA Strand Displacement and Gold Nanoparticles
}

\author{
Xin Li, ${ }^{1}$ Tao Song, ${ }^{2,3}$ Zhihua Chen, ${ }^{2,4}$ Xiaolong Shi, ${ }^{2,4}$ Congzhou Chen, ${ }^{4}$ and Zheng Zhang ${ }^{2,4}$ \\ ${ }^{1}$ Department of Obstetrics and Gynecology, Renmin Hospital of Wuhan University, Wuhan, Hubei 430060, China \\ ${ }^{2}$ College of Computer and Communication Engineering, China University of Petroleum, Qingdao, Shandong 266580, China \\ ${ }^{3}$ Faculty of Engineering, Computing and Science, Swinburne University of Technology, 93350 Kuching, Sarawak, Malaysia \\ ${ }^{4}$ Key Laboratory of Image Information Processing and Intelligent Control, School of Automation, \\ Huazhong University of Science and Technology, Wuhan, Hubei 430074, China
}

Correspondence should be addressed to Tao Song; taosong@hust.edu.cn and Xiaolong Shi; shixiaolong@hust.edu.cn

Received 6 May 2015; Revised 7 July 2015; Accepted 8 July 2015

Academic Editor: Xuping Sun

Copyright (c) $2015 \mathrm{Xin} \mathrm{Li} \mathrm{et} \mathrm{al.} \mathrm{This} \mathrm{is} \mathrm{an} \mathrm{open} \mathrm{access} \mathrm{article} \mathrm{distributed} \mathrm{under} \mathrm{the} \mathrm{Creative} \mathrm{Commons} \mathrm{Attribution} \mathrm{License,} \mathrm{which}$ permits unrestricted use, distribution, and reproduction in any medium, provided the original work is properly cited.

DNA or gene signal detection is of great significance in many fields including medical examination, intracellular molecular monitoring, and gene disease signal diagnosis, but detection of DNA or gene signals in a low concentration with instant visual results remains a challenge. In this work, a universal fast and visual colorimetric detection method for DNA signals is proposed. Specifically, a DNA signal amplification "circuit" based on DNA strand displacement is firstly designed to amplify the target DNA signals, and then thiol modified hairpin DNA strands and gold nanoparticles are used to make signal detection results visualized in a colorimetric manner. If the target DNA signal exists, the gold nanoparticles aggregate and settle down with color changing from dark red to grey quickly; otherwise, the gold nanoparticles' colloids remain stable in dark red. The proposed method provides a novel way to detect quickly DNA or gene signals in low concentrations with instant visual results. When applied in real-life, it may provide a universal colorimetric method for gene disease signal diagnosis.

\section{Introduction}

Biocomputing refers to the field of exploiting biological macromolecules or organisms to implement relatively standard methods of computation [1-5]. In recent years, computing with programmable DNA molecules has become a promising research topic, and lots of pioneering conceptual works contributed to the field, such as helical molecular programming approach [6], DNA self-assembly for computation $[7,8]$, molecular computing methods to improve the accuracy of insertion site analysis in tumors [9], and tabletop molecular communications with chemical signals [10]. One of the widely used technologies in DNA programming is DNA strand displacement [11].

DNA strand displacement technology was proposed as an in vitro isothermal and enzyme-free DNA amplification technique $[11,12]$. During the past decades, it has become a popular studied branch of DNA programming. The most notable merit of DNA strand displacement is that during its process all DNA strands interact with one another automatically and cannot "cross talk" with other unrelated strands by "carefully" sequence design. The technique is selective to the recognition of DNA sequence [13] and has been used to detect DNA signals [14-19]. With DNA strand displacement, some biomolecular signal processing systems have been developed, such as enzyme-free nucleic acid logic circuits [20], genetic programming and evolvable molecular machines [21], performing logic computation of Hopfield network autoassociative memory with DNA strands [22], kinetically controlled self-assembly of DNA oligomers [23], and logic gates with "DNA neurons" [24].

A DNA molecular system seems to be functioning unit with DNA strands having particular biological functions. In the system, information is encoded and stored in form of DNA strands, and each of DNA strands performs its own reactions independently. The output DNA strands in certain reaction can be taken as input of other reactions, which in some sense can process information like functioning 


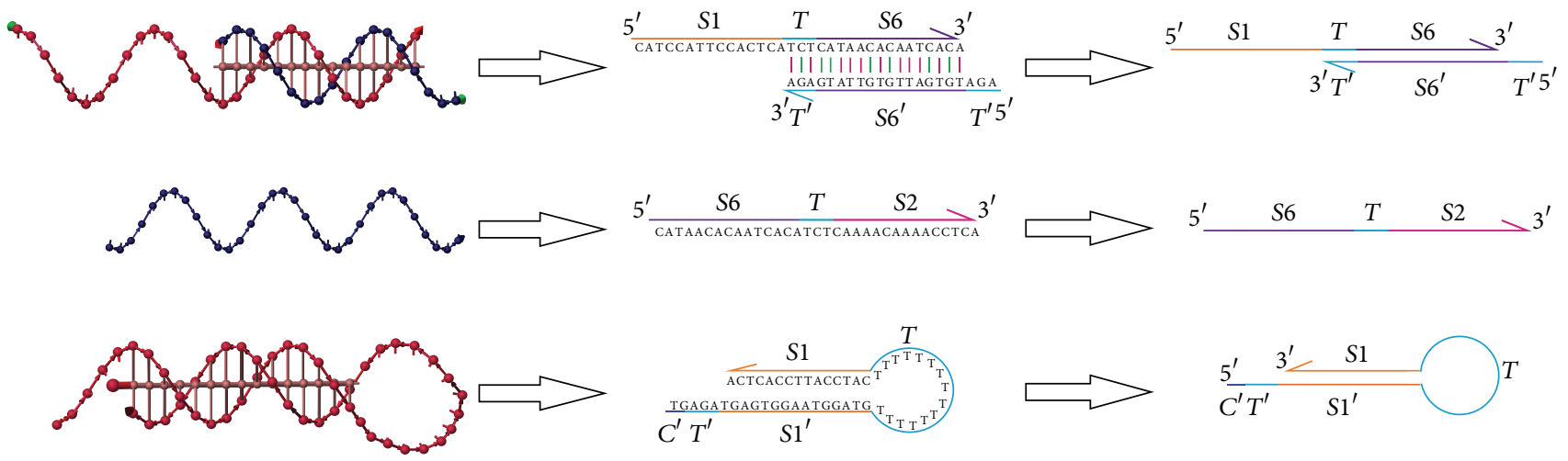

FIGURE 1: Representation of DNA strands and domains with functional segment of oligonucleotides.

unit performing chain reactions. With this fact, detection of specific DNA signals is crucial for DNA molecular systems. Before detecting target DNA signals, it is customary to amplify the target DNA signals before detection. Plenty of studies have been contributed to amplify DNA signals in DNA molecular systems [25-31]. However, detection of target DNA signals in low concentrations and with instant visualized detection result remains a challenging problem.

In this work, we propose a universal and fast visualized colorimetric method to detect DNA signals. Specifically, a DNA signal amplification "circuit" based on DNA strand displacement is firstly developed to amplify the target DNA signals (in low concentrations). After that, thiol modified hairpin DNA strands and gold nanoparticles are used for visualizing the detection results in a colorimetric manner. If target DNA signal exists, specific output DNA strands can be produced, with which the thiol modified hairpin DNA strands and gold nanoparticles cannot combine such that gold nanoparticles aggregate and settle down with color changing from dark red to grey quickly; if no target DNA signal exists, gold nanoparticles colloid keeps stable and remains in color dark red.

Experimental observations and results show that our method performs well in detecting DNA signals, and also the detection result can be visualized in a colorimetric manner. The proposed method provides a novel way to detect quickly DNA signals in low concentrations with instant visual results. For further application, it provides a universal colorimetric method for gene disease signal diagnosis.

\section{Methods}

In this section, we introduce the structure of DNA signal amplification "circuit" with DNA strand displacement, and then the visualized colorimetric method for DNA signal (in a low concentration) detection is described in detail.

2.1. DNA Signal Amplification "Circuit". It starts by introducing the way to represent DNA strands, and then the basic reactions used in DNA signal amplification "circuit" are introduced.
DNA strands are represented by straight lines with arrows that denote the direction from $5^{\prime}$ to $3^{\prime}$, as shown in Figure 1. A specific segment of oligonucleotides is defined as a "domain" to make clear the comprehension of DNA hybridization, branch migration, and dissociation. Each domain is represented by a straight line of specific color and length, such as domains $S 1, S 2, T, T^{\prime}, S 6, S 6^{\prime}$, and $C$ in Figure 1.

The domains labeled with the letter " $S$ " are "recognition domains," and the ones with label " $T$ " or " $C$ " are the "toehold domains." For any domain $\alpha$, we use $\alpha$ to represent its complementary domain, where $\alpha$ can be the label of any domain. For example, domain S6 is complementary with domain $S 6^{\prime}$.

In DNA signal amplification "circuit," there are four main types of strands, that is, "Input," "Output," "Gate," and "Fuel." DNA signal amplification "circuit" is based on the principle of toehold-mediated DNA strand displacement. It starts from a single-stranded DNA binding to the uncovered toehold domain by its own toehold domain and then allowing the migration of the prehybridized recognition domain and adhesion of incoming recognition domain. With the "circuit," the single-stranded DNA strand (DNA signal) can be replaced by the quondam bound single-stranded DNA; see $[32,33]$. There are two reactions in DNA signal amplification "circuit."

One reaction is the seesawing reaction, whose schematic diagram is shown in Figure 2.

This reaction happens when "Input" strands talk with "Gate" strands adhering to the Input-Gate complex. Initially, "Input" strand hybridizes to the extended toehold domain of "Gate" strand ( $T^{\prime}$ domain on the rightmost), and domain S6 of "Input" strand squeezes out that of "Gate" complex such that "Output" strand will be released and Input-Gate complex is produced at the same time. After Input-Gate complex is produced, "Fuel" strand will hybridize to the uncovered toehold domain of the Input-Gate complex and release "Input" strand again and "Waste" strands. With the reaction, it forms a catalytic cycle, by which "Fuel" strand can be consumed with producing "Output" strand, while the amount of "Input" strands keeps changeless. In this way, "Output" strand can be produced with consumption of "Fuel" strands, but without any reduction of "Input" strands. 


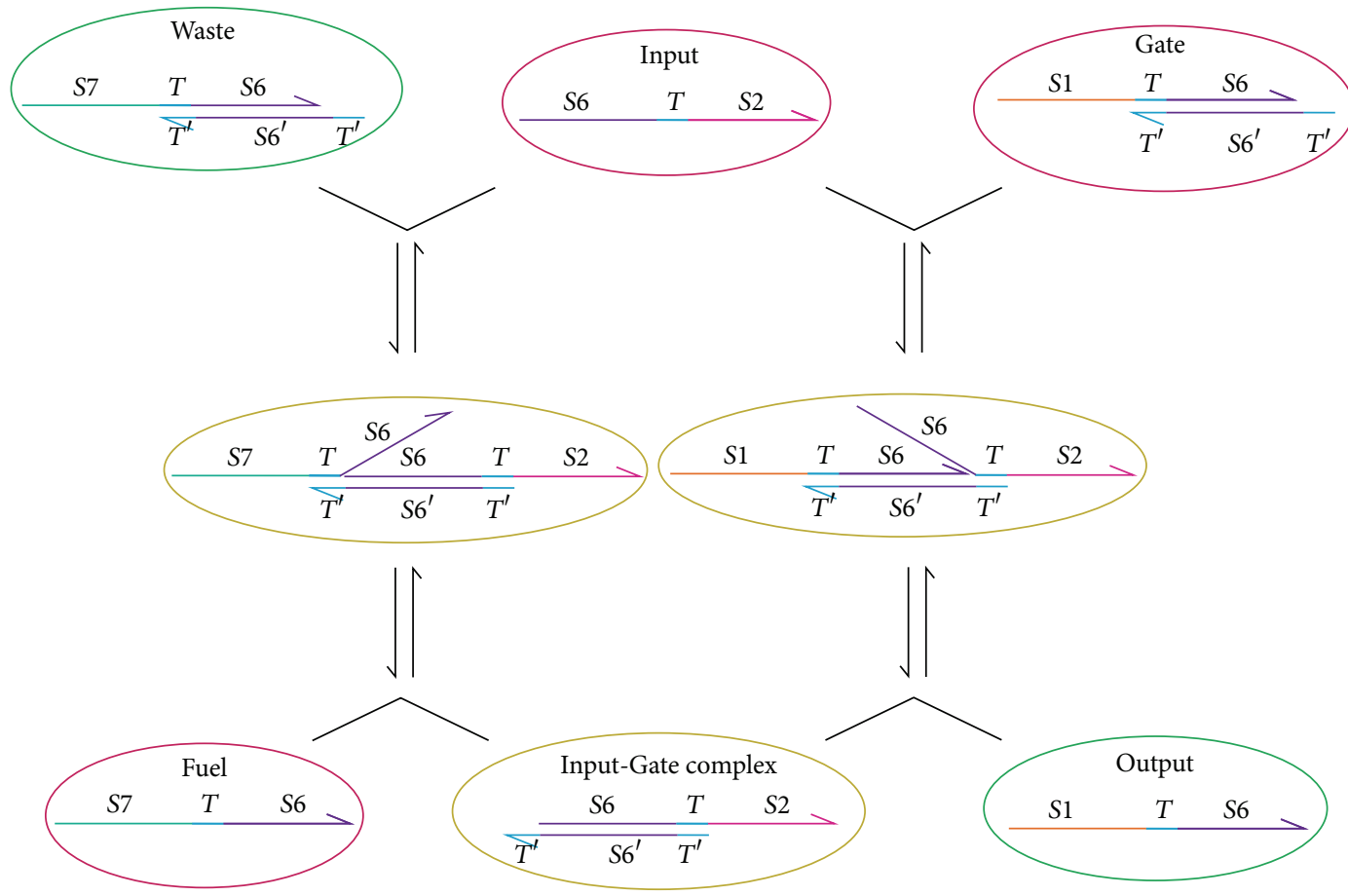

FIgURE 2: The schematic diagram of seesawing reaction.

The second reaction, that is, the reporting reaction, takes place if and only if "Output" strand has been released in seesawing reaction. When "Output" strand is released, it will adhere to the "Reporter" complex via its toehold, starting the migration and displacement. Domain $S 1$ of "Output" strand will hybridize with domain $S 1^{\prime}$ of "Reporter" strand. In this way, hairpin structure of "Reporter" strand will be forced to unfold producing the Output-Report complex, which is the resulting strand by amplifying "Input" strands.

With the two basic reactions, DNA signal amplification "circuit" can be built as shown in Figure 3. When target DNA signal "Input" strand is present, a seesawing reaction occurs immediately to release "Output" strand; meanwhile, "Fuel" strand hybridizes to the intermediary complex (Input-Gate complex) to release "Input" strands and the "Waste" strands. In this way, the "Input" strand can be produced as soon as it is consumed, so the seesawing reaction does not reduce the amount of "Input" strand. It is noted that the volume of "Fuel" strand should be several times as much as that of "Input" strand, which will promote seesawing reaction circle towards the direction of producing "Outputs" strand. If no "Input" strand is added to "circuit," then no "Output" strand can be released.

\subsection{The Visualized Colorimetric DNA Signal Detection} Method. It is known that gold nanoparticles could aggregate and precipitate in a solution of high concentration [34-39]. If gold nanoparticles cannot be connected with DNA through thiol group, they will aggregate and settle down with color changing from dark red to grey with adding salt solution; otherwise, they remain stable and in color dark red. With

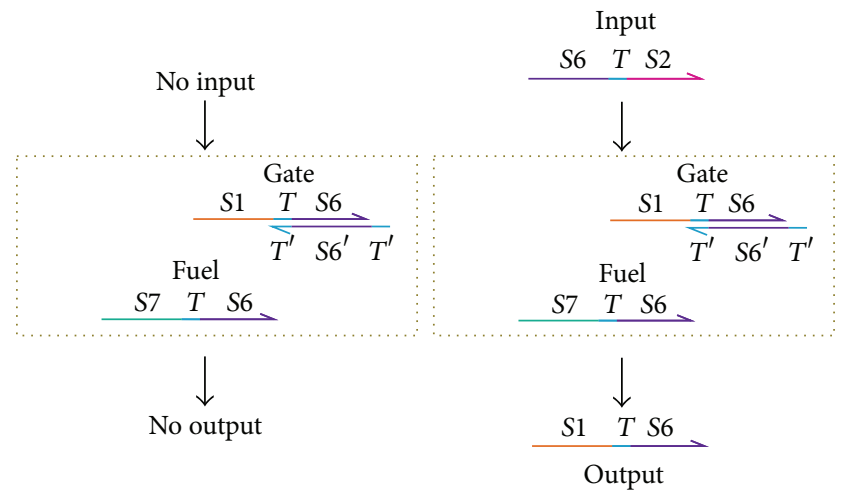

FIgURE 3: DNA signal amplification "circuit."

this fact, we develop a visualized colorimetric DNA signal detection method with aggregation and precipitation of gold nanoparticles.

A "Reporter" strand (see Figure 4) is hereby designed, whose $5^{\prime}$ side is connected with a thiol group. The schematic diagram of the visualized colorimetric DNA signal detection method is depicted in Figure 5.

If "Output" strand cannot be produced, which means no target "Input" strand exists, then thiol group of "Reporter" will bind to gold nanoparticles to prevent them from aggregating. In this case, gold nanoparticles will remain stable in dark red. The schematic diagram of the process is given in Figure 5(a).

If "Output" strand can be produced, which means target "Input" strand exists, then the thiol group of "Reporter" will 
TABLE 1: Sequences and domains with all sequences starting from $5^{\prime}$ end to $3^{\prime}$ end.

\begin{tabular}{lcc}
\hline Primer & Domains & Sequence \\
\hline P1 & $S 6+T+S 2$ & CATAACACAATCACA + TCT + CAAAACAAAACCTCA \\
P2 & $S 7+T+S 6$ & CAACATATCAATTCA + TCT + CATAACACAATCACA \\
P3 & $T^{\prime}+S 6^{\prime}+T^{\prime}$ & AGA + TGTGATTGTGTTATG + AGA \\
P4 & $S 1+T+S 6$ & CATCCATTCCACTCATC + TCT + CATAACACAATCACA \\
P5 & $C^{\prime}+T^{\prime}+S 1^{\prime}+S 1$ & TG + AGATGA + GTGGAATGGATG + TTTTTTTTTTTTTT + CATCCATTCCACTCA \\
\hline
\end{tabular}
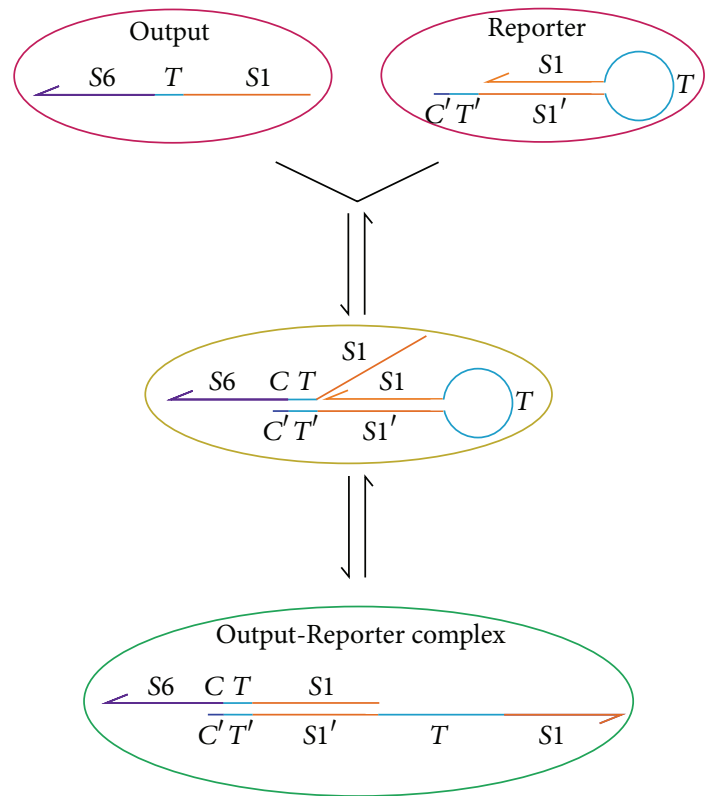

FIGURE 4: The schematic diagram of reporting reaction.

be forced to unfold such that "Reporter" cannot bind to gold nanoparticles; see Figure 5(b). In this case, gold nanoparticles aggregate and settle down quickly changing color from dark red to grey.

With the explanations above, the fast colorimetric method for DNA signal detections can be concluded as follows. If the target DNA signal (even in a low concentration) exists, it will be firstly amplified by the amplification "circuit," and the magnified signal can adhere to "Reporter" complex resulting in the failure of connection between thiol group and gold nanoparticles. When salt solution is added, gold nanoparticles aggregate and settle down with solution color changing quickly from dark red to grey. If the target DNA signals is inexistent, "Output" strand will not be released such that the bare toehold of "Reporter" allows connection between thiol group and gold nanoparticles. When the salt solution is added, gold nanoparticles colloid remains stable with the solution in color dark red.

\section{Experimental Results}

\subsection{Materials}

3.1.1. DNA Strands Preparation. All DNA strands were acquired from Shanghai Sangon Co. Ltd. Disulfide functionalized DNA strands were purified through high-performance
TABle 2: Components of "Input," "Output," “Gate," and "Fuel."

\begin{tabular}{lccc}
\hline ID & Formation & Function & Concentration \\
\hline C1 & P1 & Input & $5 \mu \mathrm{M}$ \\
C2 & P2 & Fuel & $5 \mu \mathrm{M}$ \\
C3 & P3, P4 & Gate & $5 \mu \mathrm{M}$ \\
C4 & P5 & Output & $10 \mu \mathrm{M}$ \\
\hline
\end{tabular}

liquid chromatography (HPLC). Unmodified DNA strands were also purified through HPLC. After being chemically synthesized and shipped as powder, DNA oligonucleotides were suspended in Milli-Q water (Millipore) and stored at $4^{\circ} \mathrm{C}$ at $\sim 100 \mu \mathrm{M}$. The sequences of the oligonucleotides are listed in Table 1 for ease of reference. Besides, $\mathrm{HAuCl}_{4} \cdot 3 \mathrm{H}_{2} \mathrm{O}$, trisodium citrate, and sodium chloride were also purchased from Shanghai Sangon Co. Ltd.

3.1.2. Gold Nanoparticles Preparation. Gold nanoparticles were prepared by the citrate reduction of $\mathrm{HAuCl}_{4} .100 \mathrm{~mL}$ ultrapure water, $2 \mathrm{~mL}$ auric chloride acid (0.5\%), and $10 \mathrm{~mL}$ trisodium citrate $(1 \%)$ are added to $250 \mathrm{~mL}$ conical flask and shook up. The mixture is heated with high heat in microwave oven for 1 minute and with medium heat for 5 minutes. Finally the gold nanoparticles colloid is cooled down at room temperature and examined by TEM (Transmission Electron Microscope). The obtained picture is shown in Figure 6. The TEM result shows that gold nanoparticles spread evenly with $\sim 20 \mathrm{~nm}$ diameters.

3.1.3. Amplification "Circuit" Formation. In the DNA signal amplification "circuit," there are four kinds of strands "Input," "Output," "Gate," and "Fuel." Their formations and concentrations are shown in Table 2. The "Gate" is formed in a course of slow annealing. All the components are stored at $4^{\circ} \mathrm{C}$.

3.2. Experimental Observations. The scheme of DNA signal amplification and colorimetric detection of gold nanoparticles is shown in Figure 7. There are two test tubes in DNA signal amplification reaction, which are labeled by $\mathrm{T} 1$ and T2, respectively; see Figure 7(a). DNA signal amplification "circuit" is constructed with $5 \mu \mathrm{L}$ "Gate" and $5 \mu \mathrm{L}$ "Fuel" and put into each of test tubes $\mathrm{T} 1$ and $\mathrm{T} 2$.

$1 \mu \mathrm{L}$ target DNA signal "Input" strand is added to T2, while the same amount of ultrapure water is added to T1 for comparison (see Figure 7(a)). Disulfide functionalized "Reporter" is added to both of two test tubes 0.5 hour later. Tubes TA and TB (with gold nanoparticles colloids) are kept 


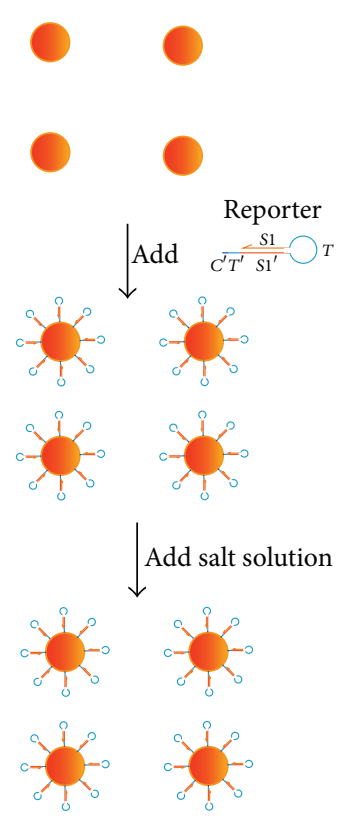

(a)

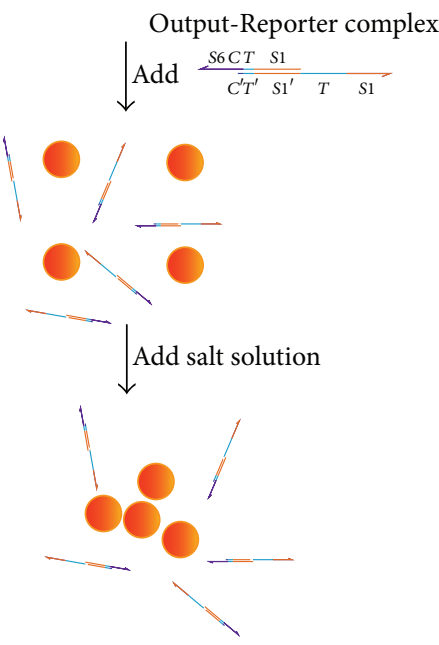

(b)

FIGURE 5: The schematic diagram of the visualized colorimetric DNA signal detection method.

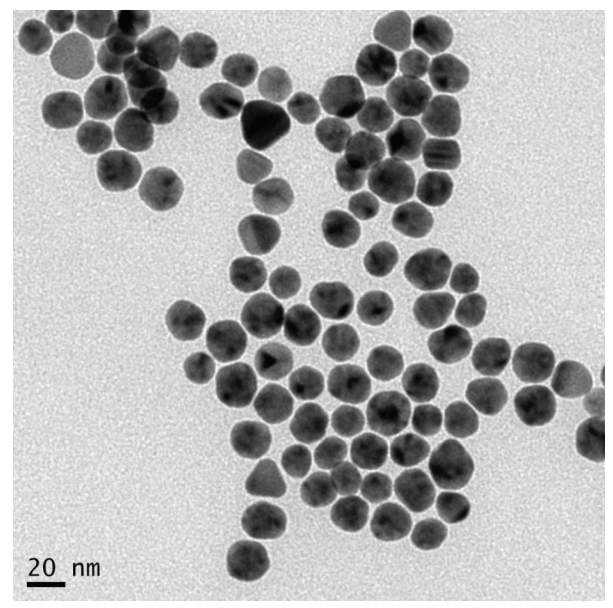

FIGURE 6: TEM image of pure gold nanoparticles.

for 22 hours for sufficient attachment between thiol groups and gold nanoparticles. After 0.5 hour, we add $3 \mu \mathrm{L}$ solution from tube $\mathrm{T} 1$ to $\mathrm{TA}$ and add $3 \mu \mathrm{L}$ solution from tube $\mathrm{T} 2$ to $\mathrm{TB}$, where tubes TA and TB are with $20 \mu \mathrm{L}$ gold nanoparticles colloid (see Figure $7(\mathrm{~b})$ ). Eventually, $0.5 \mu \mathrm{L}$ salt solution is added to each of tubes TA and TB.

The results of signal amplification of native PAGE (polyacrylamide gel electrophoresis) and visible detection results are shown in Figure 8.

In Figure 8(a), PAGE results of DNA signal amplification "circuit" are given. From the first line to the fourth, it indicates admixture of "Reporter" and "Output," "Reporter," amplification "circuit" without "Input," and amplification "circuit" with "Input," respectively. The PAGE results of admixture of
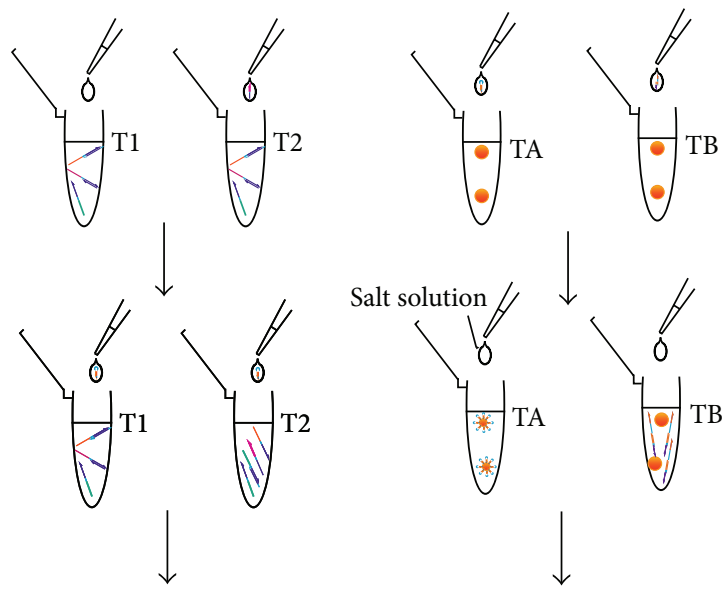

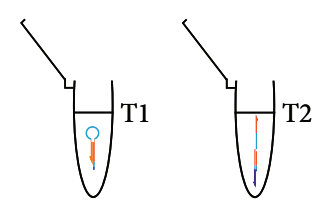

(a) (b)

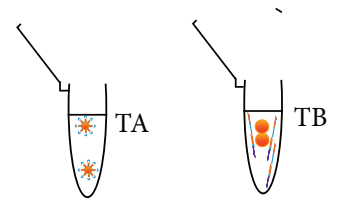

FIGURE 7: Scheme of the construction of signal amplification and colorimetric detection.

"Reporter" and "Output," "Reporter," and "Reporter" capped with thiol group are shown in Figure 8(b). Thiol group functionalized "Reporter" is added to detect the "Output" strand. It is shown in Figure 8(c) that solution in tube A is in color dark red (indicating that no target "Input" strand exists); solution in tube $\mathrm{B}$ is in color grey (indicating that 


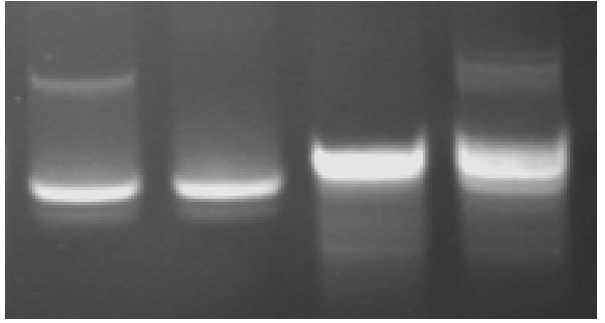

(a)

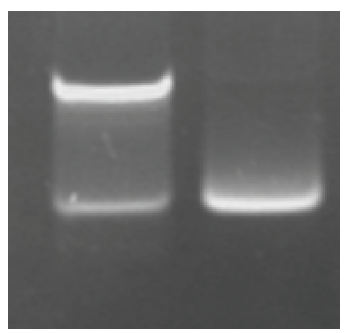

(b)

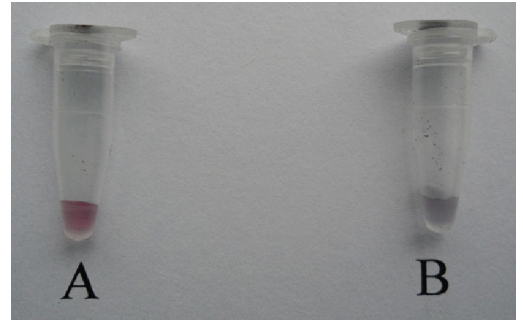

(c)

FIgURE 8: The results of signal amplification of native PAGE and visible detection results.
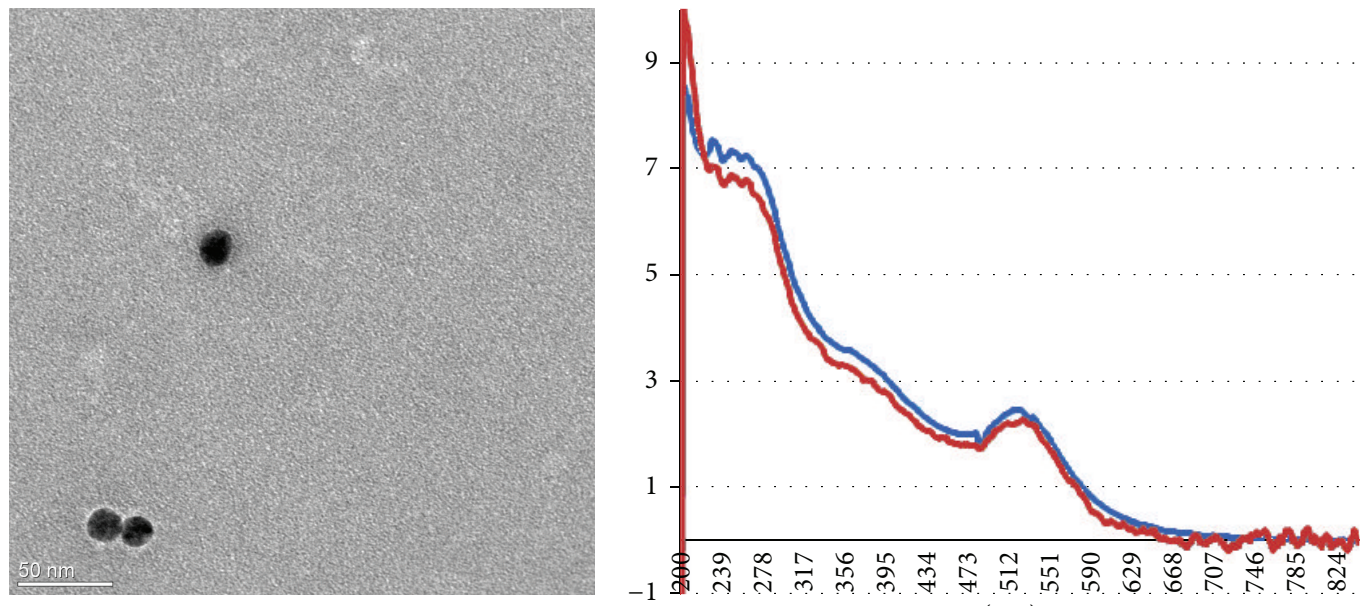

$(\mathrm{nm})$

$1.0 \mathrm{~mm}$ UV-Vis of pure GNP-20 $\mathrm{nm}$

$0.2 \mathrm{~mm}$ UV-Vis of pure GNP-20 nm

FIGURE 9: Gold nanoparticles spreading status by TEM and the UV-Vis absorption spectra by the spectra photo meter K5500 with sample diameters $1.0 \mathrm{~mm}$ (blue) and $0.2 \mathrm{~mm}$ (red).

target "Input" strand exists). The detection result can be easily visualized.

Gold nanoparticles spreading status is examined by TEM. The UV-Vis absorption spectra of the sample are detected by the spectra photo meter K5500 with sample diameter of $1.0 \mathrm{~mm}$ and $0.2 \mathrm{~mm}$. The UV-Vis absorption spectra of $20 \mathrm{~nm}$ pure gold nanoparticles with sampled diameters $1.0 \mathrm{~mm}$ (blue) and $0.2 \mathrm{~mm}$ (red) are shown in Figure 9.

Similarly, gold nanoparticles in tubes TB with target DNA signal and TA without target DNA signal are examined by TEM, and the UV-Vis absorption spectra of these samples are detected by the spectra photo meter K5500 with sample diameter of $1.0 \mathrm{~mm}$ and $0.2 \mathrm{~mm}$, respectively. The TEM pictures and UV-Vis absorption spectra of $20 \mathrm{~nm}$ gold nanoparticles in tube TA and TB are shown in Figures 10 and 11.

The TEM result of pure gold nanoparticles shown in Figure 9 indicates that the diameter of gold nanoparticle spread evenly around $20 \mathrm{~nm}$, and the UV-Vis absorption spectra result show absorption peak around $520 \mathrm{~nm}$. The TEM result of tube TA shown in Figure 10 indicates if no "Input" strand (target DNA signal) exists, gold nanoparticles cannot aggregate and settle down with $0.5 \mathrm{M} \mathrm{NaCl}$ added in the solution, and UV-Vis peak around $520 \mathrm{~nm}$ can be observed. The TEM result of tube TB is shown in Figure 11, which indicates if "Input" strand (target DNA signal) exists, it can be amplified by the amplification "circuit" and gold nanoparticles will aggregate and settle down with $0.5 \mathrm{M} \mathrm{NaCl}$ added in the solution, color changing from dark red to grey. The correspondence UV-Vis absorption spectra results show no absorption peak around $520 \mathrm{~nm}$ which confirmed the aggregation of gold nanoparticles.

3.3. Discussion. In this work, we propose a method for detection of DNA signals, where the detection results can be visualized in a colorimetric manner. Specifically, a DNA signal amplification "circuit" based on DNA strand displacement is firstly designed to amplify the target DNA signals, and then thiol modified hairpin DNA strands and gold nanoparticles are used to make signal detection results visualized in a colorimetric manner. PAGE results shown in Figures 8(a) and $8(\mathrm{~b})$ indicate good performances of the DNA signal amplification "circuit," as well as the detection results which can be visualized by the color (dark red or grey) of solution induced by aggregation or not of gold nanoparticles. We 

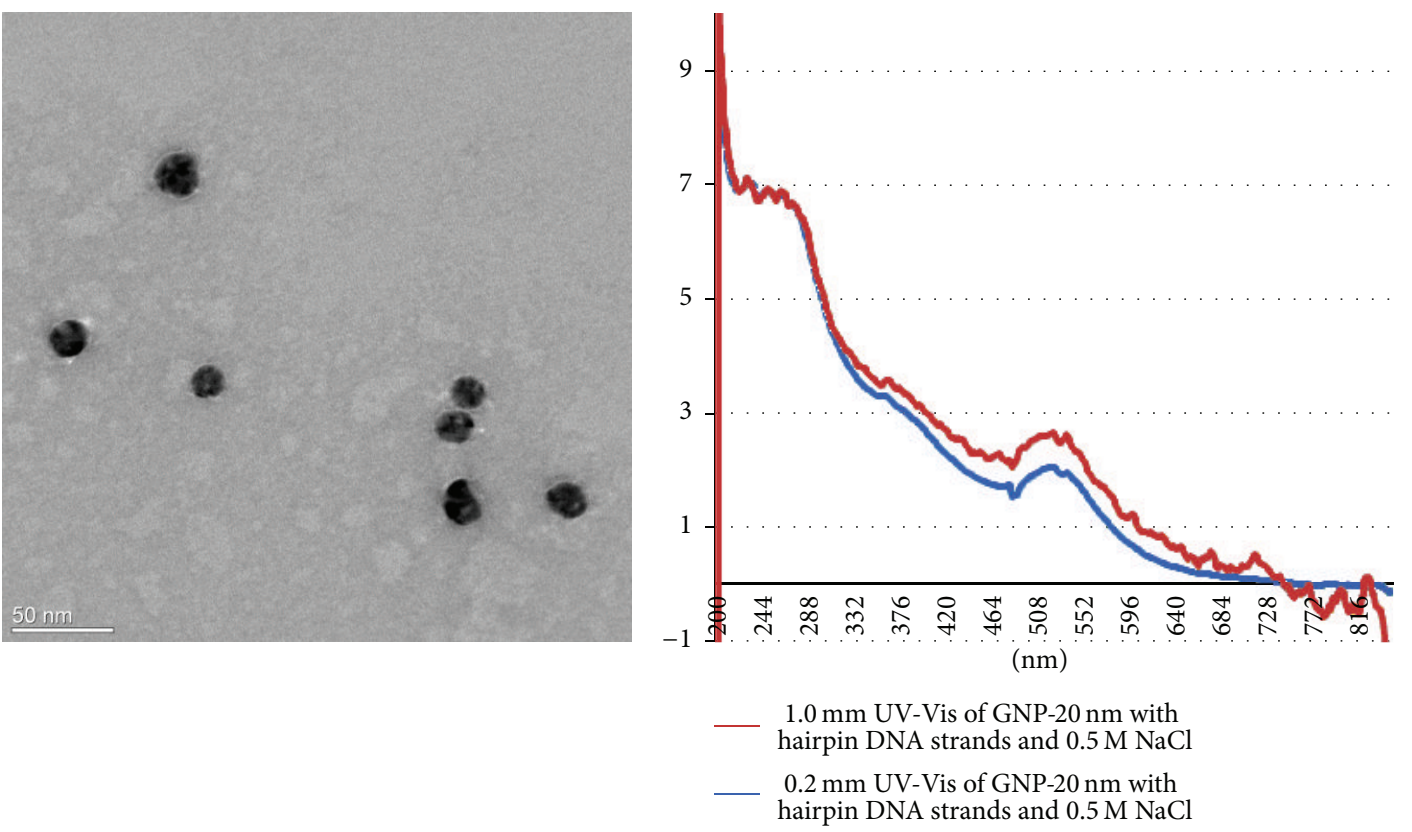

FIGURE 10: Gold nanoparticles spreading status in tube TA by TEM and the UV-Vis absorption spectra by the spectra photo meter K5500 with sample diameters $1.0 \mathrm{~mm}$ (blue) and $0.2 \mathrm{~mm}$ (red).
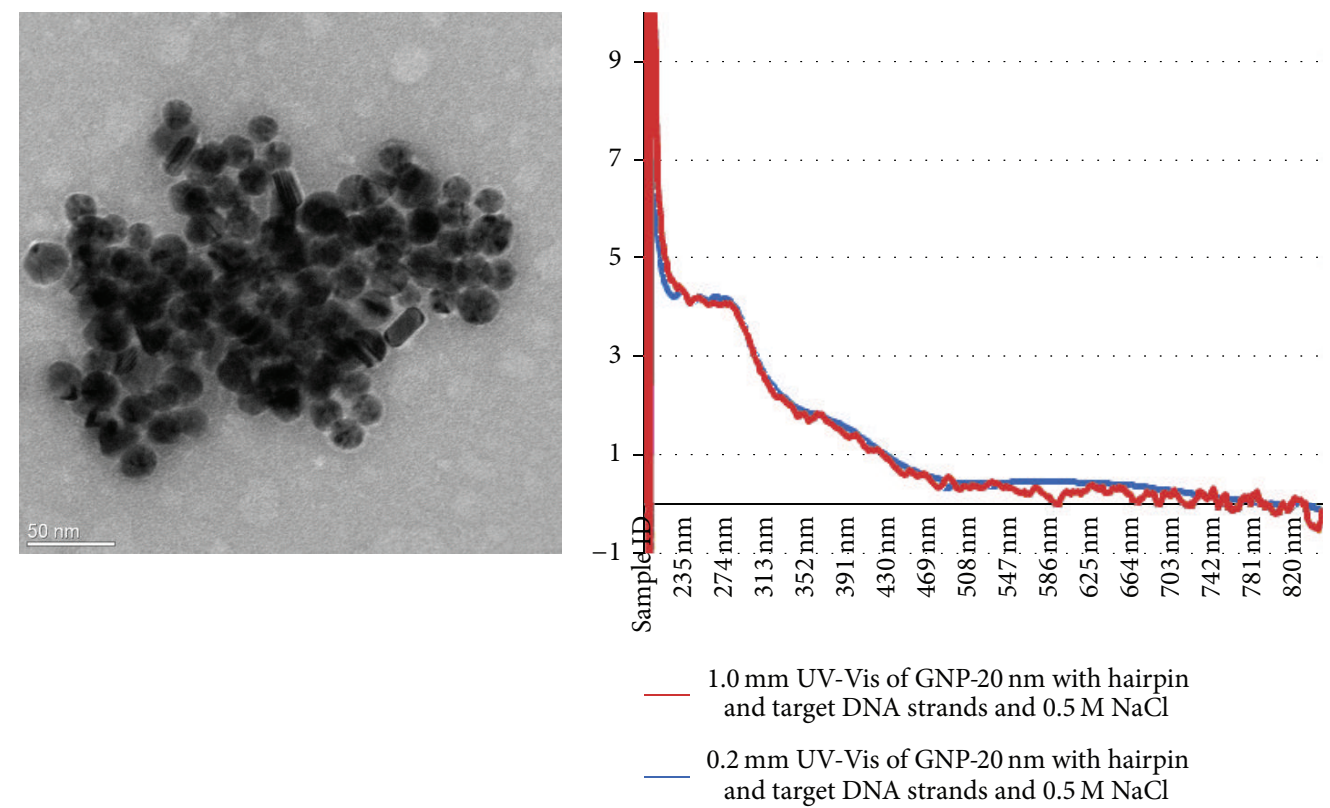

FIGURE 11: TEM picture and UV-Vis absorption spectra of $20 \mathrm{~nm}$ gold nanoparticles by the spectra photo meter K5500 with sample diameters $1.0 \mathrm{~mm}$ (blue) and $0.2 \mathrm{~mm}$ (red) in tube TB with $0.5 \mathrm{M} \mathrm{NaCl}$.

investigate the stability of the proposed method by repeating the experiment 5 times. In each experiment, we can observe the color changing as theoretically designed and can achieve the similar TEM images of gold nanoparticles and UV-Vis spectra. With this fact, it is believed that our method should be stable.

Although DNA strand displacement performs well in detecting DNA signal, there are still some problems for further research. One is the leaky reaction, in which "Output" strand can be produced even if no "Input" strand is present. Leaky reaction may cause serious consequences and could lead to logic errors [40-42]. In our method, we ameliorate the DNA strand displacement reaction by tailoring its reaction time and reducing the volume of fuel strand. Furthermore, the concentration of gate complex is selected to be $5 \mu \mathrm{M}$ in our experiment with the purpose to give a palpable result and coordinate an appropriate rate between DNA and gold nanoparticles. The volume can be further reduced to 
nM-scale to improve the sensitivity of detection enormously, since the volume of "Output" is closely related to the volume of "Gate" rather than that of "Input" strand. In this way, from the theoretical point of view, even a single "Input" strand can be detected using our strategy, but we do not verify it in the present work.

Compared to Storhoff's colorimetric DNA detection method with gold nanoparticle, our method has the merits of fluorescence-free and enzyme-free, which undergo a color change that is visually detectable [36]. Also, DNA signal amplification "circuit" with DNA strand displacement proposed in this work is more universal comparing to the formal works that nucleic acid targets are recognized by DNA-modified gold probes. This is due to the fact that in our method the target DNA signal is merely an input signal of DNA logical gate, while DNA-modified gold probes could be programmed as a constant DNA sequence with only response to the output of DNA strand displacement. Furthermore, the formal works can only deal with unamplified DNA signals, but our method has the function to DNA signal amplification.

\section{Conclusion and Future Work}

This paper developed a universal fast colorimetric method to detect DNA signal in low concentrations. A DNA signal amplification "circuit" with DNA strand displacement is firstly developed to magnify ultrasensitive and selective DNA signals, and then thiol modified hairpin DNA strands and gold nanoparticles are used to make detection results visualized in a colorimetric manner. With the DNA amplification "circuit," target DNA signal can be amplified into a signal of certain size by DNA strand displacement, which provides great convenience to subsequent detection. Besides, process of signal amplification is independent with detection.

Both qualitative and quantitative detection are achieved. It is worthy to study what will happen if "Threshold" complex constructed in [24] is added to the "circuit." In addition, the concentrations of components are set as $\mu \mathrm{M}$-scale limited by properties of gold nanoparticles, but more sensitivity is attainable when concentrations are reduced.

\section{Conflict of Interests}

The authors declare that there is no conflict of interests regarding the publication of this paper.

\section{Acknowledgments}

This work is supported by the National Science Foundations of China (Grant nos. 61272071, 61370105, 61402187, and 61425002) and Science Foundations of Hubei Province (no. 2014CFB730).

\section{References}

[1] L. M. Adleman, "Molecular computation of solutions to combinatorial problems," Science, vol. 266, no. 5187, pp. 1021-1024, 1994.
[2] P. W. K. Rothemund, N. Papadakis, and E. Winfree, "Algorithmic self-assembly of DNA Sierpinski triangles," PLoS Biology, vol. 2, no. 12, article e424, 2004.

[3] Y. Benenson, T. Paz-Elizur, R. Adar, E. Keinan, Z. Llvneh, and E. Shapiro, "Programmable and autonomous computing machine made of biomolecules," Nature, vol. 414, no. 6862, pp. 430-434, 2001.

[4] N. Hampp, "Bacteriorhodopsin as a photochromic retinal protein for optical memories," Chemical Reviews, vol. 100, no. 5, pp. 1755-1776, 2000.

[5] J. A. Stuart, D. L. Marcy, K. J. Wise, and R. R. Birge, "Volumetric optical memory based on bacteriorhodopsin," Synthetic Metals, vol. 127, no. 1-3, pp. 3-15, 2002.

[6] A. E. Rowan and R. J. M. Nolte, "Helical molecular programming," Angewandte Chemie-International Edition, vol. 37, no. 1-2, pp. 63-68, 1998.

[7] E. Winfree, F. Liu, L. A. Wenzler, and N. C. Seeman, "Design and self-assembly of two-dimensional DNA crystals," Nature, vol. 394, no. 6693, pp. 539-544, 1998.

[8] S. J. de Vries and M. Zacharias, "ATTRACT-EM: a new method for the computational assembly of large molecular machines using cryo-EM map," PLoS ONE, vol. 7, no. 12, Article ID e49733, 2012.

[9] B. T. Brett, K. E. Berquam-Vrieze, K. Nannapaneni, J. Huang, T. E. Scheetz, and A. J. Dupuy, "Novel molecular and computational methods improve the accuracy of insertion site analysis in sleeping Beauty-Induced tumors," PLoS ONE, vol. 6, no. 9, Article ID e24668, 2011.

[10] N. Farsad, W. Guo, and A. W. Eckford, “Tabletop molecular communication: text messages through chemical signals," PLoS ONE, vol. 8, no. 12, Article ID e82935, 2013.

[11] G. T. Walker, M. S. Fraiser, J. L. Schram, M. C. Little, J. G. Nadeau, and D. P. Malinowski, "Strand displacement amplification - an isothermal, in vitro DNA amplification technique," Nucleic Acids Research, vol. 20, no. 7, pp. 1691-1696, 1992.

[12] M. C. Little, J. Andrews, R. Moore et al., "Strand displacement amplification and homogeneous real-time detection incorporated in a second-generation DNA probe system, BDProbeTecET," Clinical Chemistry, vol. 45, no. 6 I, pp. 777-784, 1999.

[13] P. E. Nielsen, M. Egholm, R. H. Berg, and O. Buchardt, "Sequence-selective recognition of DNA by strand displacement with a thymine-substituted polyamide," Science, vol. 254, no. 5037, pp. 1497-1500, 1991.

[14] T. H. LaBean and H. Li, "Constructing novel materials with DNA," Nano Today, vol. 2, no. 2, pp. 26-35, 2007.

[15] E. C. Samano, M. Pilo-Pais, S. Goldberg, B. N. Vogen, G. Finkelstein, and T. H. LaBean, "Self-Assembling DNA templates for programmed artificial biomineralization," Soft Matter, vol. 7, no. 7, pp. 3240-3245, 2011.

[16] M. Pilo-Pais, S. Goldberg, E. Samano, T. H. LaBean, and G. Finkelstein, "Connecting the nanodots: programmable nanofabrication of fused metal shapes on DNA templates," Nano Letters, vol. 11, no. 8, pp. 3489-3492, 2011.

[17] X. Shi, X. Li, Z. Zhang, and J. Xu, "Improve capability of DNA automaton: DNA automaton with three internal states and tape head move in two directions," in Advances in Intelligent Computing: International Conference on Intelligent Computing, ICIC 2005, Hefei, China, August 23-26, 2005, Proceedings, Part II, vol. 3645 of Lecture Notes in Computer Science, pp. 71-79, Springer, Berlin, Germany, 2005. 
[18] X. Shi, L. Pan, and J. Xu, "General DNA automaton model with $\mathrm{R} / \mathrm{W}$ tape," in Computational Intelligence and Bioinformatics, vol. 4115 of Lecture Notes in Computer Science, pp. 258-266, Springer, Berlin, Germany, 2006.

[19] Y. Wang, P. Hu, X. Shi, and G. Cui, "DNA self-assembly for graph vertex 3-coloring problem," Journal of Computational and Theoretical Nanoscience, vol. 9, no. 12, pp. 2086-2092, 2012.

[20] G. Seelig, D. Soloveichik, D. Y. Zhang, and E. Winfree, "Enzyme-free nucleic acid logic circuits," Science, vol. 314, no. 5805, pp. 1585-1588, 2006.

[21] R. Poli, N. F. McPhee, and J. E. Rowe, "Exact schema theory and Markov chain models for genetic programming and variablelength genetic algorithms with homologous crossover," Genetic Programming and Evolvable Machines, vol. 5, no. 1, pp. 31-70, 2004.

[22] L. Qian and E. Winfree, "Scaling up digital circuit computation with DNA strand displacement cascades," Science, vol. 332, no. 6034, pp. 1196-1201, 2011.

[23] D. Lubrich, S. J. Green, and A. J. Turberfield, "Kinetically controlled self-assembly of DNA oligomers," Journal of the American Chemical Society, vol. 131, no. 7, pp. 2422-2423, 2009.

[24] X. Shi, Z. Wang, C. Deng, T. Song, L. Pan, and Z. Chen, "A novel bio-sensor based on DNA strand displacement," PLoS ONE, vol. 9, no. 10, Article ID e108856, 2014.

[25] M. L. Collins, B. Irvine, D. Tyner et al., "A branched DNA signal amplification assay for quantification of nucleic acid targets below 100 molecules/ml," Nucleic Acids Research, vol. 25, no. 15, pp. 2979-2984, 1997.

[26] M. J. Callow, S. Drmanac, and R. Drmanac, "Selective DNA amplification from complex genomes using universal doublesided adapters," Nucleic Acids Research, vol. 32, no. 2, article e21, 2004.

[27] S. Lim, O. K. Koo, Y. S. You et al., "Enhancing nanoparticlebased visible detection by controlling the extent of aggregation," Scientific Reports, vol. 2, article 456, 2012.

[28] Z. Zhang, M. B. Kermekchiev, and W. M. Barnes, "Direct DNA amplification from crude clinical samples using a PCR enhancer cocktail and novel mutants of Taq," The Journal of Molecular Diagnostics, vol. 12, no. 2, pp. 152-161, 2010.

[29] R. S. Lasken, "Genomic DNA amplification by the multiple displacement amplification (MDA) method," Biochemical Society Transactions, vol. 37, no. 2, pp. 450-453, 2009.

[30] P. Shankaranarayanan, M.-A. Mendoza-Parra, W. van Gool, L. M. Trindade, and H. Gronemeyer, "Single-tube linear DNA amplification for genome-wide studies using a few thousand cells," Nature Protocols, vol. 7, no. 2, pp. 328-339, 2012.

[31] G. Caetano-Anollés, B. J. Bassam, and P. M. Gresshoff, "DNA amplification fingerprinting using very short arbitrary oligonucleotide primers," Nature Biotechnology, vol. 9, no. 6, pp. 553557, 1991.

[32] L. Qian, E. Winfree, and J. Bruck, "Neural network computation with DNA strand displacement cascades," Nature, vol. 475, no. 7356, pp. 368-372, 2011

[33] D. Y. Zhang and E. Winfree, "Control of DNA strand displacement kinetics using toehold exchange," Journal of the American Chemical Society, vol. 131, no. 47, pp. 17303-17314, 2009.

[34] C. A. Mirkin, R. L. Letsinger, R. C. Mucic, and J. J. Storhoff, "A DNA-based method for rationally assembling nanoparticles into macroscopic materials," Nature, vol. 382, no. 6592, pp. 607609, 1996.
[35] H. Li and L. J. Rothberg, "DNA sequence detection using selective fluorescence quenching of tagged oligonucleotide probes by gold nanoparticles," Analytical Chemistry, vol. 76, no. 18, pp. 5414-5417, 2004.

[36] J. J. Storhoff, A. D. Lucas, V. Garimella, Y. P. Bao, and U. R. Müller, "Homogeneous detection of unamplified genomic DNA sequences based on colorimetric scatter of gold nanoparticle probes," Nature Biotechnology, vol. 22, no. 7, pp. 883-887, 2004.

[37] Y. Li, H. J. Schluesener, and S. Xu, "Gold nanoparticle-based biosensors," Gold Bulletin, vol. 43, no. 1, pp. 29-41, 2010.

[38] S. Y. Park, A. K. R. Lytton-Jean, B. Lee, S. Weigand, G. C. Schatz, and C. A. Mirkin, "DNA-programmable nanoparticle crystallization," Nature, vol. 451, no. 7178, pp. 553-556, 2008.

[39] S.-J. Park, A. A. Lazarides, C. A. Mirkin, P. W. Brazis, C. R. Kannewurf, and R. L. Letsinger, "The electrical properties of gold nanoparticle assemblies linked by DNA," Angewandte Chemie-International Edition, vol. 39, no. 21, pp. 3845-3848, 2000 .

[40] D. Soloveichik, G. Seelig, and E. Winfree, "DNA as a universal substrate for chemical kinetics," Proceedings of the National Academy of Sciences of the United States of America, vol. 107, no. 12, pp. 5393-5398, 2010.

[41] I. J. Nijman, M. Mokry, R. van Boxtel, P. W. Toonen, E. de Bruijn, and E. Cuppen, "Mutation discovery by targeted genomic enrichment of multiplexed barcoded samples," Nature Methods, vol. 7, no. 11, pp. 913-915, 2011.

[42] W. Fontana, "Pulling strings," Science, vol. 314, no. 5805, pp. 1552-1553, 2006. 

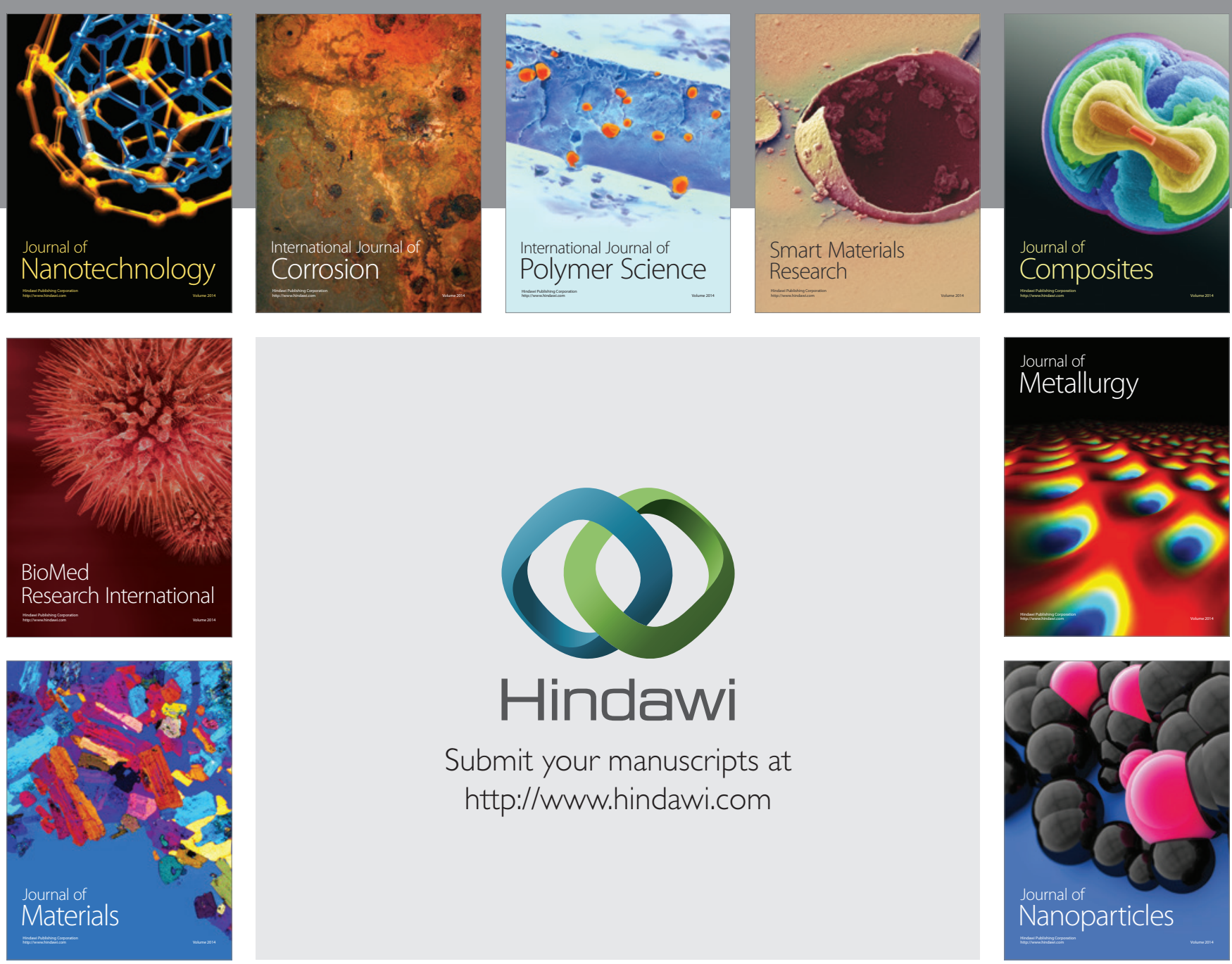

Submit your manuscripts at http://www.hindawi.com
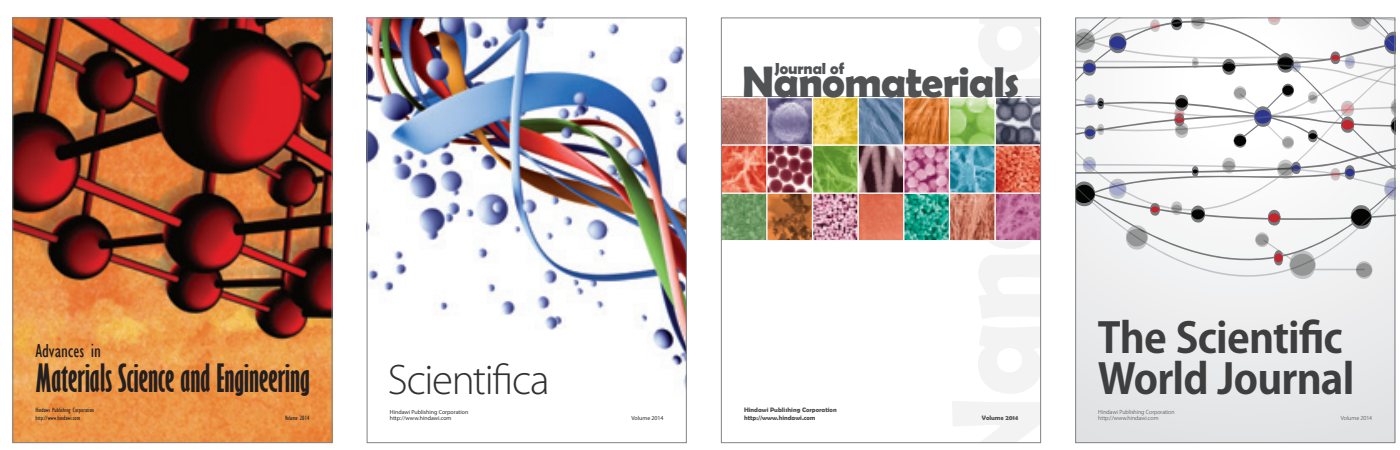

\section{The Scientific World Journal}
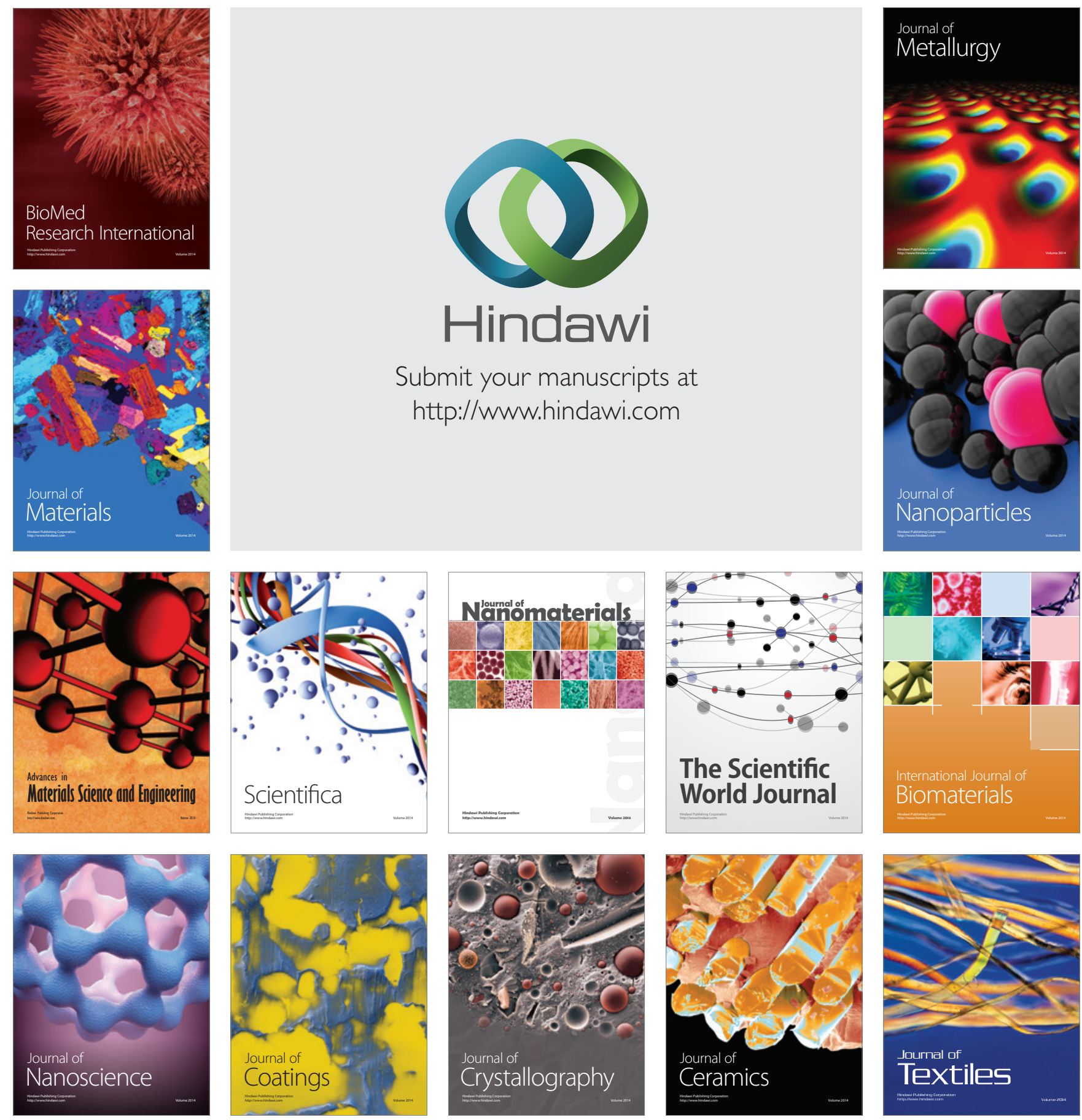